T.J. Cormwell and R.A. Perley (eds.)

\title{
A NEW METHOD OF ANALYSIS AND REDUCTION OF VLBI OBSERVATIONS
}

\author{
E. F. ARIAS
}

Facultad de Ciencias Astronómicas y Geofísicas (UNLP), CONICET, IERS(C/B)

Paseo del Bosque s/n, 1900 La Plata, Argentina.

\section{S. DE BIASI}

Facultad de Ciencias Astronómicas y Geofísicas (UNLP), CONICET Paseo del Bosque s/n, 1900 La Plata, Argentina.

\begin{abstract}
In this paper we develope an inertial system of coordinates introducing a new observable: the arc between a pair of radio sources. We shall discuss how the conditional equation involving all the parameters is written in diferent systems when the observations are performed with a two and a three-element interferometers.
\end{abstract}

\section{INTRODUCTION}

In the classical algorithm of analysis of VLBI observations, the equatorial coordinates of the radio sources, the coordinates of the stations, the Earth Orientation Parameters (EOP) and, eventually, the corrections to the position of the celestial pole given by the IAU 1976/1980 models are simultaneously adjusted.

We have explored a new method of constructing a celestial frame independently of the terrestrial frame and of the Earth's orientation in space. The new strategy of observation consists on "ideally" observing two extragalactic radio sources simultaneously with two pairs of radiotelescopes, each one at the extremities of a VLBI baseline. On the basis of the classical VLBI observables - the delay and the delay rate- we have constructed a new observable: the arc between a pair of sources. A set of these new observables leads to spherical coordinates of the radio sources relative to an arbitrary plane and origin.

We have started with the simplest case, where the delay and the delay rate are entirely due to the VLBI geometry. Then, we have considered all their components. In all the cases, the effects of polar motion, UT1-UTC, precession and nutation are neglected.

\section{THE METHOD}

In the first approach, we assume that the delay and the delay rate are due to the VLBI geometry only and we write the equations of observations in a geocentric equatorial frame. 
The baseline vector $\vec{B}$ remains fixed in a terrestrial frame while the unit vector $\vec{\sigma}$ in the direction of the radio source remains fixed in a celestial frame. Be $S(O, x, y, z)$ a geocentric frame fixed in space, with the $x$-axis near the direction of the equinox and the $z$-axis in the direction of the rotation axis of the Earth. $S^{\prime}\left(O, x^{\prime}, y^{\prime}, z^{\prime}\right)$ is a geocentric frame fixed in the Earth, with the $x^{\prime}$-axis in the direction of the Greenwich meridian; as we are neglecting the effects of polar motion, precession and nutation, the $z$-axis of both frames are coincident.

The declination $\delta_{B}$ of $\vec{B}$ remains constant in the frame fixed in space, but its right ascension $\alpha_{B}$ changes with time as the right ascension of the Greenwich meridian does.

The arc $A$ between two sources of coordinates $\left(\alpha_{1}, \delta_{1}\right),\left(\alpha_{2}, \delta_{2}\right)$ can be written:

$$
\cos A=\sin \delta_{1} \sin \delta_{2}+\cos \delta_{1} \cos \delta_{2} \cos \left(\alpha_{1}-\alpha_{2}\right)
$$

For each radio source $i$ the technique of VLBI determines the delay $\tau_{i}$ and the delay rate $\dot{\tau}_{i}$, wich can be written in terms of the radio source and the baseline coordinates. The observation of the pair of sources gives four equations. Combining them with eq. (1) the arc $A$ adopts the form:

$$
\cos A=f\left(\tau_{1}, \dot{\tau}_{1}, \tau_{2}, \dot{\tau}_{2}, \sec ^{2} \delta_{B}, \sec ^{4} \delta_{B}, B, \partial \alpha_{G}(t) / \partial t\right)
$$

We have eliminated the radio sources coordinates $\left(\alpha_{1}, \delta_{1}\right),\left(\alpha_{2}, \delta_{2}\right)$ and the right ascension $\alpha_{B}$ of the baseline, but its declination $\delta_{B}$ is still present. The dependence on the declination of the baseline makes this expression useless. Our goal is to eliminate all the parameters except the fundamental VLBI observables $\tau$ and $\dot{\tau}$. It is probable that the equatorial system we have used to write our equations is not the best adapted to our analysis.

As the arc is independent of the system adopted, we can choose a set of arbitrary directions in space. We can define a new orthogonal frame associated to the baseline and to the angular rotation velocity of the Earth $\bar{\Omega}$. The direction of the new axes $\hat{\imath}_{1}, \hat{\imath}_{2}, \hat{\imath}_{3}$ are given by

$$
\begin{aligned}
& \hat{\imath}_{1}=\hat{e} \\
& \hat{\imath}_{2}=\hat{\omega} \wedge \hat{\imath}_{1} \\
& \hat{\imath}_{3} \perp \hat{\imath}_{2} \perp \hat{\imath}_{1}
\end{aligned}
$$

where $\hat{e}$ and $\hat{\omega}$ are the unit vectors of $\vec{B}$ and $\vec{\Omega}$ respectively.

Although the modules of the vectors $\vec{B}, \vec{\Omega}$ and the arc $A$ are unknown we can adopt a priori values $B_{0}, \Omega_{0}, A_{0}$ and include in the unknowns their dimensionless corrections $\Delta B, \Delta \Omega, \Delta A$.

Writing the observables $\tau_{1}, \dot{\tau}_{1}, \tau_{2}, \dot{\tau}_{2}$ in the new reference system we obtain the differential expresion:

$$
\sin A_{0} \Delta A=\cos A_{0}+C_{1}+C_{2} \Delta B+C_{3} \Delta \Omega
$$

where we have consider only the first orders in the small corrections $\Delta B, \Delta \Omega$, $\Delta A$, as they are $\approx 10^{-8}$. The coefficients $C_{i}$ in eq (3) depend on the known parameters $B_{0}, \Omega_{0}, \tau_{i}, \dot{\tau}_{i}$. 
Let us consider now a more realistic expression of the delay and the delay rate. If $p(t)$ takes into account the effects of the propagation medium and $q_{0}, q$ are the clock constants, they can be written as:

$$
\begin{aligned}
& \tau=\tau_{g}+p(t)+q_{0}+q \Delta t \\
& \dot{\tau}=\dot{\tau}_{g}+\dot{p}(t)+q
\end{aligned}
$$

where $\tau_{g}$ is the geometric component of the delay.

Under these conditions, the correction $\Delta A$ to the arc can be expressed as:

$$
\sin A_{0} \Delta A=\cos A_{0}+C_{1} \Delta B+C_{2} Q_{0}+C_{3} Q+C_{4} \Delta \Omega+C_{5} P+C_{6} \dot{P}+C_{7}
$$

up to the first order in the small quantities $\Delta B, \Delta \Omega, p, q, q_{0}, \dot{p}$.

The theoretical precission of this method is given by the order of the terms neglected. The largest contribution is due to the term in $\Delta \Omega^{2}$, that is the correction to the module of the angular rotation velocity of the Earth. Adopting standard values of the known parameters it is aproximately $0.0004^{\prime \prime}$

Observing the pair of sources with a three-element interferometer we can define a reference system independently of the Earth rotation vector. This new system will be attached to the unit vectors $\hat{e}_{1}, \hat{e}_{2}$ in the directions of two of the baselines in the form:

$$
\begin{aligned}
& \hat{\imath}_{1}^{\prime}=\hat{e}_{1} \\
& \hat{\imath}_{2}^{\prime}=\hat{e}_{1} \wedge \hat{e}_{2} \\
& \hat{\imath}_{3}^{\prime} \perp \hat{\imath}_{2}^{\prime} \perp \hat{\imath}_{1}^{\prime}
\end{aligned}
$$

The observed delays are (first subscript denotes baseline and second denotes source):

$$
\begin{aligned}
& \tau_{11}=-\frac{\vec{B}_{1} \cdot \hat{e}_{1}}{c}+p_{11}(t)+q_{01}+q_{1} \Delta t \\
& \tau_{12}=-\frac{\vec{B}_{1} \cdot \hat{e}_{2}}{c}+p_{12}(t)+q_{01}+q_{1} \Delta t \\
& \tau_{21}=-\frac{\vec{B}_{2} \cdot \hat{e}_{1}}{c}+p_{21}(t)+q_{02}+q_{2} \Delta t \\
& \tau_{22}=-\frac{\vec{B}_{2} \cdot \hat{e}_{2}}{c}+p_{22}(t)+q_{02}+q_{2} \Delta t
\end{aligned}
$$

From these last four equations we can obtain an expression for the correction $\Delta A$ to the arc wich is analogous to eq (4). A new parameter is addeed, the relative positions of baselines $D$, wich can be written as $D=$ $D_{0}(1+\Delta D)$, being $D_{0}$ an adopted value and $\Delta D$ an unknown correction of the order $10^{-8}$.

We have analysed the particular case in wich the observations are conducted with one interferometer in the East-West direction and the other in the North-South direction $\left(D_{0}=90^{\circ}\right)$. Finally:

$$
\begin{aligned}
\Delta A \sin A_{0}= & \cos A_{0}+C_{0}+C_{1} \Delta B_{1}+C_{2} \Delta B_{2}+C_{3} \Delta D \\
& +C_{4} P_{11}+C_{5} P_{12}+C_{6} P_{21}+C_{7} P_{22}+C_{8} Q_{01}+C_{9} Q_{02} \\
& +C_{10} Q_{1} \Delta T+C_{11} Q_{2} \Delta T
\end{aligned}
$$

where $C_{i}=C_{i}\left(B_{01}, B_{02}, D_{0}, \tau_{11}, \tau_{12}, \tau_{21}, \tau_{22}\right)$. 
The largest terms neglected are:

$$
\begin{aligned}
& \frac{2 c^{2}}{B^{2}} \tau \Delta B q_{0} \approx 10^{-7 \prime \prime} \\
& \frac{4 c^{4}}{B^{4}} \tau^{3} \Delta B q_{0} \approx 4 \times 10^{-8 \prime \prime}
\end{aligned}
$$

\section{CONCLUSION}

We have shown that it is possible to obtain a set of equations of observations independently of the baseline orientation and the vector of angular rotation velocity of the Earth. Adopting a reference system attached to the baseline(s) we can construct a new observable, the arc between a pair of radio sources simultaneously observed.

Observing $K$ pairs of sources, eq (5) gives $K$ equations of observation with $K$ unknowns $\Delta A$ and three more unknowns $\Delta B_{1}, \Delta B_{2}, \Delta D$. It is preferable to parametrize the system with a minimum number of unknowns transforming the $K$ corrections to the arcs $\Delta A$ into corrections $(\Delta \phi, \Delta \epsilon)$ to arbitrary spherical coordinates of the radio sources.

In this new system the origins are fixed by adopting a priori values for three coordinates i.e. $\phi, \epsilon$ of a source and $\epsilon$ of another one. In the new parameters, eq (5) becomes:

$$
\sin A_{0} \Delta A=f_{1} \Delta \epsilon_{1}+f_{2} \Delta \epsilon_{2}+f_{3}\left(\Delta \phi_{1}-\Delta \phi_{2}\right)
$$

Five radio sources are enough to obtain a solution. However, we prefer to have a superabundant system to solve it with a least squares fit.

The theoretical precission of the method, estimated from the terms neglected in the developments, is at the level of $0.0004^{\prime \prime}$ when the observations are performed with a two-element interferometer and the reference system is attached to the unique baseline and to the direction of the vector of rotation velocity of the Earth. When the reference system is defined only by two baseline vectors (case of the three-element interferometer), and only the delay $\tau$ is considered in the expression of the arc, the theoretical precission is $10^{3}$ times higher.

\section{REFERENCES}

Dravskikh,A.F.,Krasinsky,G.A.,Finkelstein,A.M. 1975 Soviet Astron. Lett. Vol 1, 3, 110.

Dravskikh,A.F.,Finkelstein,A.M.,Krasinsky,G.A. 1975 Astroph. and Space Science, $\underline{38}, 255$.

Dravskikh,A.F.,Finkelstein,A.M.,Kreinovich, V.Ja. 1978 IAU Colloquium 48 , 143. 